\title{
Who Are the Undiagnosed?
}

\section{Disparities in Hypertension Diagnoses in Vulnerable Populations}

\author{
Margaret Meador, MPH; Joy H. Lewis, DO, PhD; R. Curtis Bay, PhD; Hilary K. Wall, MPH; \\ Chanaye Jackson, MPH
}

This study builds upon a project that developed clinical criteria to identify undiagnosed hypertension patients "hiding in plain sight" (HIPS) by examining patient characteristics to understand whether there are disparities in hypertension diagnosis. We examined electronic health record demographic data for patients identified by the HIPS criteria and subgroups at 3 Missouri health centers. Identified patients who returned for a follow-up visit and were subsequently diagnosed with hypertension tended to be older, black/African American, uninsured, and classified as having obesity. Younger, white, healthy weight females were less likely to be diagnosed. These findings point to exploring possible biases/other nonclinical factors in hypertension diagnosis.

Key words: health centers, health disparities, population health, social determinants of health, undiagnosed hypertension

ARDIOVASCULAR DISEASE accounts for 1-in-7 US health care dollars spent-almost $\$ 1$ billion per day ${ }^{1}$-and accounts for 800000

\begin{abstract}
Author Affiliations: National Association of Community Health Centers, Bethesda, Maryland (Mss Meador and Jackson); Department of Public Health, School of Osteopathic Medicine in Arizona, A. T. Still University, Mesa (Dr Lewis); Department of Interdisciplinary Health Sciences, Arizona School of Health Sciences, A.T. Still University, Mesa (Dr Bay); and Division for Heart Disease and Stroke Prevention, Centers for Disease Control and Prevention, Atlanta, Georgia (Ms Wall).

The authors wish to thank Michael Rakotz, MD, FAHA, FAAFP, for providing technical assistance in informing the development of the algorithm used to identify participants, and advising the project. The authors also wish to thank J. Aaron Allgood, DO, for his clinical insights and suggestions for this article. In addition, the authors wish to thank the following organizations for their dedicated work in this project:

- Affinia Healthcare, St Louis, Missouri

- Jordan Valley Community Health Center, Springfield, Missouri

- Myrtle Hilliard Davis Comprehensive Health Centers, St Louis, Missouri

- Missouri Primary Care Association
\end{abstract}

This project was funded by US Federal Award Identification Number (US) ЗU80T00223 from the Centers for Disease Control and Prevention. The findings and conclusions in this report are those of the authors and do not necessarily reflect the official position of the Centers for Disease Control and Prevention.

Written work prepared by employees of the Federal Government as part of their official duties is, under the U.S. Copyright Act, a "work of the United States Government" for which copyright protection under Title 17 of the United States Code is not available. As such, copyright does not extend to the contributions of employees of the Federal Government.

The authors declare no conflicts of interest.

Correspondence: Margaret Meador, MPH, National Association of Community Health Centers, 7501 Wisconsin Ave Ste 1100W, Bethesda, MD 20814 (mmeador@nachc.org).

DOI: $10.1097 / \mathrm{FCH} .0000000000000242$ deaths every year, representing almost a third of US deaths. ${ }^{2}$ By 2030 , direct medical costs linked to cardiovascular diseases are projected to exceed $\$ 818$ billion annually, while lost productivity costs could surpass $\$ 275$ billion. $^{2}$ Many cardiovascular events resulting in emergency department visits, hospitalizations, and deaths are preventable with early detection, lifestyle modifications, and treatment. ${ }^{1,3,4}$

Hypertension is a major risk factor for cardiovascular disease. ${ }^{5}$ Hypertension is second only to cigarette smoking as a preventable cause of death for any reason. ${ }^{3}$ Current data suggest over 14 million people are not aware of their hypertension and are, consequently, not taking medication for it or engaged in other interventions to control their blood pressure. $^{6}$

Recently, efforts to leverage electronic health records (EHRs) and other health information technology to identify patients with undiagnosed chronic conditions to ensure timely and appropriate diagnosis and treatment have increased..$^{7-13} \mathrm{New}$ guidelines for blood pressure management recommend using health information technology to identify patients with elevated blood pressure who are not diagnosed with hypertension. ${ }^{5}$ Further understanding the characteristics of those with undiagnosed hypertension is an important step to help clinical care teams improve targeting and tailoring of interventions for diagnosing hypertension.

\section{BACKGROUND}

Hypertension prevalence increases substantially with age and varies greatly by sex, racial/ethnic group, and comorbid conditions., ${ }^{5,16}$ Among adults with a usual source of care, younger adults who meet the clinical criteria for hypertension are less likely than other adults to be diagnosed with hypertension and less likely to be diagnosed in a timely manner. $^{13}$ 
Access to health care has traditionally played a role in timely diagnosis of chronic disease. ${ }^{17}$ Adults without health insurance and/or a usual source of care are less likely than those with insurance to receive routine preventive care, including screening for high blood pressure. ${ }^{18,19}$ Males aged 15 to 50 years infrequently seek preventive care services, regardless of insurance status, ${ }^{20}$ which could increase their rates of undiagnosed hypertension.

Diagnostic inertia for hypertension-when a patient without known hypertension is classified as normal by the medical staff when presenting with elevated blood pressure-is also very real and may occur as frequently as 1 -in-3 cases of high blood pressure. ${ }^{21}$ It has also been suggested that provider expectations for who should have hypertension may be a factor in who is diagnosed with hypertension. ${ }^{12}$

To address the problem of undiagnosed hypertension, a recent project funded by the Centers for Disease Control and Prevention and led by the National Association of Community Health Centers used a quality improvement approach to develop clinical criteria to identify potentially undiagnosed hypertension patients "hiding in plain sight" (HIPS) at 10 federally qualified health centers. These criteria were then formatted into an algorithm (Figure 1) that was embedded into clinical decision support mechanisms to identify patients who might have undiagnosed hypertension both at the point of care and using population health management strategies. After implementation of algorithm-based interventions, $31.9 \%$ of patients identified by the HIPS algorithm who completed a blood pressure evaluation were then formally diagnosed with hypertension. ${ }^{12}$

While age and barriers to accessing care have clearly surfaced as determinants of appropriate hy-

Two sets of clinical criteria were used together, corresponding to clinical stages of HTN based on 2014 hypertension guidelines, ${ }^{1}$ (stage 1: systolic BP [SBP] measurement between $140 \mathrm{mmHg}$ and $159 \mathrm{mmHg}$ or diastolic BP [DBP] measurement between $90 \mathrm{mmHg}$ and $99 \mathrm{mmHg}$; stage 2: SBP measurement $\geq 160 \mathrm{mmHg}$ or DBP

measurement $\geq 100 \mathrm{mmHg}^{*}$ :

Stage 1 Criteria. Patients 18 to 85 years old without a diagnosis of hypertension (documented as an ICD-9-CM [International Classification of Diseases, Ninth Revision, Clinical Modification] 17 assessment of 401-405) who have SBP or DBP measurements consistent with the definition of stage 1 hypertension at two separate medical visits, including the most recent visit, during the past 12 months. Exclusions: pregnancy, end-stage renal disease (ESRD),

Stage 2 Criteria. Patients 18 to 85 years old without a diagnosis of hypertension who have a SBP or DBP measurement consistent with the definition of stage 2 hypertension at any one medical visit during the past 12 months. Exclusions: pregnancy, ESRD.

-NOTE: Using the recent 2017 Hypertension Guidelines, ${ }^{6}$ Stage 1 is defined as SBP 130 $130 \mathrm{mmHg}$ or DBP $80-89$, and Stage 2 is defined as SBP $\geq 140 \mathrm{mmHg}$ or DBP $\geq 90 \mathrm{mmHg}$

Figure 1. Clinical criteria for the HIPS algorithm. HIPS indicates hiding in plain sight. pertension diagnosis in existing research, ${ }^{17-21}$ this study builds on the HIPS project results by attempting to understand whether there are other patient characteristics associated with undiagnosed hypertension. Identifying these additional characteristics and potential disparities could help explain whether hypertension diagnoses are being delayed or missed in a systematic fashion affecting certain groups; if so, this knowledge and awareness could then be incorporated into care team training to reduce disparities in hypertension identification and care.

\section{METHODS}

The institutional review board at A.T. Still University's Kirksville College of Osteopathic Medicine in Kirksville, Missouri, granted exempt status to this study. This article was informed by the SQUIRE Guidelines V2.0.22

\section{Study population and data collection}

We identified patients using an algorithm-based query of EHRs on January 31, 2015. The HIPS algorithm, the details of which are published elsewhere, evaluated whether patients had elevated blood pressure measurements documented in their EHR in the past 12 months (Figure 1). ${ }^{12}$

From this point forward, patients identified by the algorithm as meeting clinical criteria for hypertension and therefore being potentially undiagnosed with hypertension will be referred to as HIPS patients. We used EHR data to examine demographic characteristics of adult HIPS patients as of January 31,2015 . These patients were tracked longitudinally for 17 months (February 1, 2015, to June 30, 2016). Using this HIPS population, we aimed to understand the characteristics of patients who fell into 2 categories (Figure 2): (1) patients we could reach, which includes patients identified by the HIPS algorithm who returned for a visit between February 1, 2015, and June 30, 2016, during which blood pressure was assessed and (2) patients we could not reach, which includes those who were identified by the HIPS algorithm but did not return for a visit between February 1, 2015, and June 30, 2016. Those who could be reached were then dichotomized into (A) patients for whom blood pressure was assessed and hypertension was confirmed and formally diagnosed and (B) patients who were not diagnosed within the study time frame.

\section{Setting and participants}

This study involved 3 multisite safety net health center organizations in Missouri that provide primary care to underserved areas or populations. These organizations comprise a subset of the original 


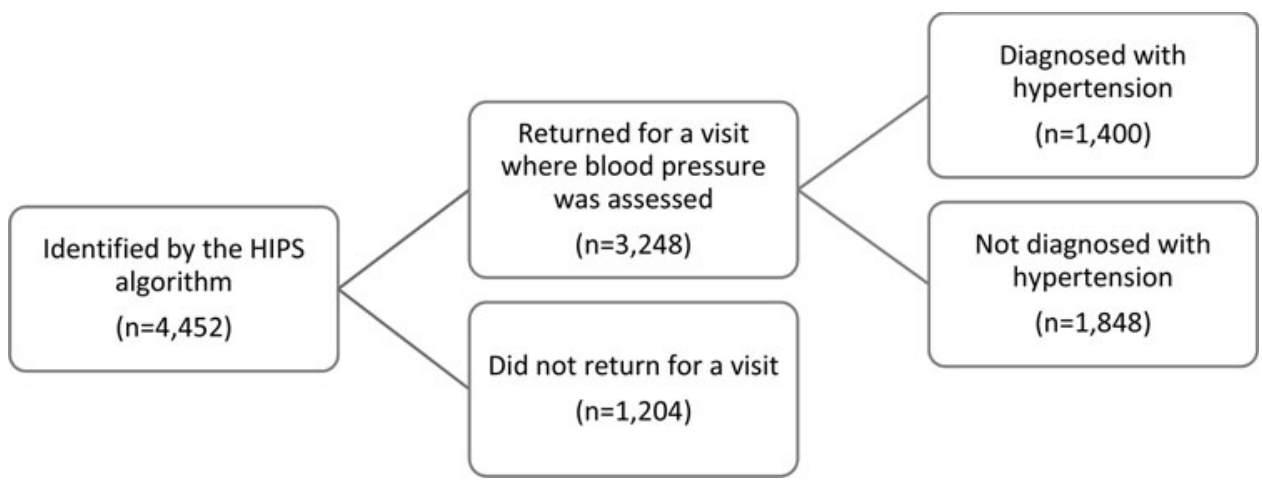

Figure 2. Patients identified by the HIPS algorithm by return visit and hypertension diagnosis status. HIPS indicates hiding in plain sight.

10 organizations in the HIPS project that were able to follow patients longitudinally. ${ }^{12}$ Two health centers' physical clinic locations were urban $(7$ sites total) with patient populations who were predominantly black/African American. A third health center had both suburban and rural sites (4 sites total), with a predominantly non-Hispanic white population. In total, these centers served 51393 adult patients aged 18 to 85 years at the inception of this study (Table 1).

\section{Data analysis}

A descriptive analysis was conducted to summarize demographic characteristics of the population of patients identified by the HIPS algorithm as well as features of subgroups delineated by return visit and hypertension diagnosis with regard to age, sex, race (white, black/African American), insurance status (insured/uninsured), housing status (homeless/housing insecure) at the time of the study, and obesity status using body mass index (BMI), defined as obesity (BMI $\geq 30 \mathrm{~kg} / \mathrm{m}^{2}$ ), overweight (BMI $\left.25-29.9 \mathrm{~kg} / \mathrm{m}^{2}\right)$, and healthy weight (BMI $<25.0 \mathrm{~kg} / \mathrm{m}^{2}$ ). Insufficient data were available to explore analysis of ethnicity, smaller racial groups (eg, Asian/Pacific Islander and Native American/Alaska Native), poverty level, and other comorbidities.

Bivariate and multivariable statistical analyses were performed to identify patient characteristics associated with patients identified by the HIPS

\section{TABLE 1. Health Center Demographic Characteristics ${ }^{a, b}$}

\begin{tabular}{|c|c|c|c|}
\hline Demographics & Health Center 1 & Health Center 2 & Health Center 3 \\
\hline Number of sites & 4 & 4 & 3 \\
\hline Adult population (18-85 y old), $\mathrm{n}$ & 22553 & 14697 & 14143 \\
\hline $\begin{array}{l}\text { Self-reported urbanicity of care } \\
\text { delivery locations }\end{array}$ & Urban & Suburban/rural & Urban \\
\hline \multicolumn{4}{|l|}{ Race $^{c}, \%$} \\
\hline Non-Hispanic white & 17.40 & 89.12 & 3.17 \\
\hline Black/African American & 69.92 & 5.21 & 96.31 \\
\hline \multicolumn{4}{|l|}{ Ethnicity, \% } \\
\hline Hispanic/Latino & 11.11 & 5.13 & 0.25 \\
\hline Best served in another language, \% & 9.08 & 2.55 & 0.77 \\
\hline Patients $\leq 100 \%$ of poverty level, $\%$ & 95.79 & 70.98 & 90.08 \\
\hline Patients $\leq 200 \%$ of poverty level, $\%$ & 99.73 & 96.39 & 99.24 \\
\hline Uninsured, \% & 42.43 & 23.75 & 25.90 \\
\hline Housing insecure, \% & 8.23 & 1.87 & 19.23 \\
\hline
\end{tabular}

aSource for all data: HRSA Health Center Data 2016 (as of December 31, 2016), available: https://bphc.hrsa.gov/uds/datacenter.aspx; except for number of sites and adult population, which were reported directly from health centers during the project (as of June 30, 2016).

${ }^{b}$ Raw Uniform Data System (UDS) data were not available for analysis, so overall row totals could not be generated.

${ }^{\mathrm{C}}$ In the UDS, race and ethnicity are presented separately but are not completely mutually exclusive. Data on Hispanic/Latino ethnicity among the black/African American population are not separated out and, therefore, there may be some overlap. 
algorithm. Among this group, we calculated bivariate and multivariable risk ratios to identify characteristics associated with those who did and did not return for a visit where blood pressure was assessed. ${ }^{23}$ Finally, we examined characteristics of patients who had a visit and were subsequently diagnosed with hypertension compared with those who had a visit but did not receive a diagnosis. Data were analyzed using SPSS version 24.

\section{RESULTS}

\section{Descriptive data}

Overall, 4452 adults, ages 18 to 85 years, were identified by the HIPS algorithm in the 3 health centers on January 31, 2015. Females constituted $61.9 \%$ and blacks/African Americans represented 73.0\% of those identified. Nearly half were uninsured $(49.3 \%)$. The majority of people were younger than 65 years; $36.1 \%$ aged 50 to 64 years and $32.9 \%$ aged 36 to 49 years, and $22.9 \%$ aged 18 to 35 years.
Over half of those identified were classified as obese $(55.0 \%)$, and $25.3 \%$ were classified as overweight (Table 2).

Among these HIPS patients, 3248 (73.0\%) returned for a visit where blood pressure was assessed and $1204(27.0 \%)$ did not return during the evaluation time frame. Of those who returned, $1400(43.1 \%)$ were diagnosed with hypertension. Patient characteristics for each group are described in Table 2.

\section{Predictors of return for a follow-up visit}

Demographic variables significantly associated with whether HIPS patients returned for a visit where blood pressure was assessed included sex, race, age, health insurance status, and obesity.

Table 3 provides the results of bivariate and multivariable analyses of patient characteristics tested against whether they returned $(\mathrm{n}=3248)$ or failed to return $(\mathrm{n}=1204)$. All predictors were entered simultaneously for the multivariable analysis.

\section{TABLE 2. Characteristics of Adult Patients Identified by the HIPS algorithm by Return Visit Status and Hypertension Diagnosis Status}

\begin{tabular}{|c|c|c|c|c|c|}
\hline \multirow[b]{2}{*}{ Variable/Category } & \multicolumn{3}{|c|}{ Patients Identified by the HIPS Algorithm } & \multicolumn{2}{|c|}{ HTN Diagnosis (Returned Group) } \\
\hline & $\begin{array}{c}\text { All } \\
\mathrm{n}(\%)\end{array}$ & $\begin{array}{c}\text { Returned } \\
\text { n (\%) }\end{array}$ & $\begin{array}{c}\text { Did Not Return } \\
\mathrm{n}(\%)\end{array}$ & $\begin{array}{l}\text { Diagnosed } \\
\text { n (\%) }\end{array}$ & $\begin{array}{c}\text { Not Diagnosed } \\
n(\%)\end{array}$ \\
\hline Total & 4452 & 3248 (73.0) & $1204(27.0)$ & $1400(43.1)$ & 1848 (56.9) \\
\hline \multicolumn{6}{|l|}{ Sex } \\
\hline Male & $1695(38.1)$ & $1145(67.6)$ & $550(32.4)$ & $529(46.2)$ & $616(53.8)$ \\
\hline Female & 2757 (61.9) & 2103 (76.3) & $654(23.7)$ & $871(41.4)$ & $1232(58.6)$ \\
\hline \multicolumn{6}{|l|}{ Race } \\
\hline White & 1202 (27.0) & 833 (69.3) & $369(30.7)$ & $290(34.8)$ & $543(65.2)$ \\
\hline Black/African American & $3250(73.0)$ & $2415(74.3)$ & 835 (25.7) & $1110(46.0)$ & $1305(54.0)$ \\
\hline \multicolumn{6}{|l|}{ Age, y } \\
\hline $18-35$ & $1020(22.9)$ & 719 (70.5) & 301 (29.5) & $201(28.0)$ & $518(72.0)$ \\
\hline $36-49$ & $1466(32.9)$ & $1058(72.2)$ & $408(27.8)$ & $456(43.1)$ & $602(56.9)$ \\
\hline $50-64$ & $1608(36.1)$ & $1205(74.9)$ & $403(25.1)$ & $614(51.0)$ & $591(49.0)$ \\
\hline$\geq 65$ & $358(8.0)$ & $266(74.3)$ & $92(25.7)$ & $129(48.5)$ & $137(51.5)$ \\
\hline \multicolumn{6}{|l|}{ Health insurance } \\
\hline Insured & 2256 (50.7) & $1596(70.7)$ & 660 (29.3) & $610(38.2)$ & $986(61.8)$ \\
\hline Uninsured & 2196 (49.3) & $1652(75.2)$ & $544(24.8)$ & $790(47.8)$ & 862 (52.2) \\
\hline \multicolumn{6}{|l|}{ Housing } \\
\hline Home & 4287 (96.3) & $3132(73.1)$ & 1155 (26.9) & $1355(43.3)$ & $1777(56.7)$ \\
\hline Housing insecure & $165(3.7)$ & 116 (70.3) & $49(29.7)$ & $45(38.8)$ & $71(61.2)$ \\
\hline \multicolumn{6}{|l|}{ Obesity (BMl in $\mathrm{kg} / \mathrm{m}^{2}$ ) } \\
\hline Normal weight (BMI <25) & 877 (19.3) & $618(70.5)$ & $259(29.5)$ & $224(36.2)$ & $394(63.8)$ \\
\hline Overweight (BMI 25-29.9) & $1126(25.3)$ & $766(68.0)$ & $360(32.0)$ & $314(41.0)$ & $452(59.0)$ \\
\hline Obesity (BMI $\geq 30$ ) & $2449(55.0)$ & $1864(76.1)$ & 585 (23.9) & $862(46.2)$ & 1002 (53.8) \\
\hline
\end{tabular}

Abbreviations: BMI, body mass index; HIPS, hiding in plain sight; HTN, hypertension. 


\section{TABLE 3. Predictors of Return Visits Where Blood Pressure Was Assessed}

\begin{tabular}{|c|c|c|c|c|c|c|}
\hline \multirow[b]{3}{*}{ Variable } & \multicolumn{6}{|c|}{ Analysis of Return $(n=3248)$ vs Did Not Return $(n=1204)$} \\
\hline & \multicolumn{3}{|c|}{ Bivariate } & \multicolumn{3}{|c|}{ Multivariable } \\
\hline & $\mathbf{R R}$ & $95 \% \mathrm{Cl}$ & $P$ Value & $\mathbf{R R}$ & $95 \% \mathrm{Cl}$ & $P$ Value \\
\hline \multicolumn{7}{|l|}{ Sex } \\
\hline Male & 1.0 & & & 1.0 & & \\
\hline Female & 1.13 & $1.09-1.17$ & $<.001$ & 1.13 & $1.08-1.17$ & $<.001$ \\
\hline \multicolumn{7}{|l|}{ Race } \\
\hline White & 1.0 & & & & & \\
\hline Black/African American & 1.07 & $1.03-1.12$ & .001 & 1.05 & $1.01-1.10$ & .022 \\
\hline \multicolumn{7}{|l|}{ Age, y } \\
\hline $18-35$ & 1.0 & & & 1.0 & & \\
\hline $36-49$ & 1.02 & $0.97-1.08$ & .364 & 1.02 & $0.97-1.02$ & .431 \\
\hline $50-64$ & 1.06 & $1.01-1.12$ & .014 & 1.08 & $1.03-1.14$ & .002 \\
\hline$\geq 65$ & 1.05 & $0.98-1.13$ & .156 & 1.12 & $1.04-1.21$ & .004 \\
\hline \multicolumn{7}{|l|}{ Health insurance } \\
\hline Insured & 1.0 & & & 1.0 & & \\
\hline Uninsured & 1.06 & $1.03-1.10$ & $<.001$ & 1.08 & $1.04-1.12$ & $<.001$ \\
\hline \multicolumn{7}{|l|}{ Housing } \\
\hline Home & 1.0 & & & & & \\
\hline Housing insecure & 0.96 & $0.87-1.06$ & .455 & 1.00 & $0.90-1.10$ & .937 \\
\hline \multicolumn{7}{|l|}{ Obesity (BMl in $\mathrm{kg} / \mathrm{m}^{2}$ ) } \\
\hline Normal weight (BMI <25) & 1.0 & & & 1.0 & & \\
\hline Overweight (BMI 25-29.9) & 0.97 & 0.91-1.02 & .239 & 0.96 & $0.91-1.02$ & .173 \\
\hline Obesity (BMI $\geq 30$ ) & 1.08 & $1.03-1.13$ & .002 & 1.06 & $1.01-1.11$ & .018 \\
\hline
\end{tabular}

Abbreviations: $\mathrm{BMI}$, body mass index; $\mathrm{Cl}$, confidence interval; $\mathrm{RR}$, risk ratio.

Multivariable analyses revealed that females were $13 \%$ more likely than males to return for a followup visit $(P<.001)$ and black/African American patients $5 \%$ more likely to return than white patients $(P=.022)$. Age was also predictive using the multivariable analysis, with patients aged 50 to 64 years $8 \%$ more likely to return $(P=.002)$ and patients 65 years or older $12 \%$ more likely to return $(P<$ .004) than patients aged 18 to 34 years. Multivariable risk ratios also showed that uninsured patients were $8 \%$ more likely to return than those with insurance $(P<.001)$ and that weight also factored into return rates, with patients who had obesity $6 \%$ more likely to return than patients who were not obese or overweight $(P=.018)$.

\section{Predictors of hypertension diagnosis}

Table 4 provides the results of bivariate and multivariable analyses of patient characteristics tested against whether a subsequent hypertension diagnosis was made $(\mathrm{n}=1400)$ or not $(\mathrm{n}=1848)$ for HIPS patients who returned for a visit where blood pressure was assessed. Variables significantly associated with subsequent diagnosis included sex, race, age, health insurance status, and obesity.

A bivariate risk ratio analysis revealed that females were $10 \%$ less likely than males to be diagnosed with hypertension $(P=.008)$. When controlling for other factors, this difference by sex disappeared $(P=.894)$. However, racial differences in hypertension diagnosis were found when controlling for other factors. Blacks/African Americans who were identified by the HIPS algorithm were $39 \%$ more likely to receive a hypertension diagnosis after a return visit to assess blood pressure when compared with whites $(P<.001)$. Age also remained a significant predictor of hypertension diagnosis using a multivariable analysis; patients were more than twice as likely if they were 65 years or older $(P<.001)$ versus $95 \%$ more likely $(P<.001)$ if they were aged 50 to 64 years to be diagnosed with hypertension when compared with patients aged 18 to 35 years.

Being uninsured was predictive of receiving a hypertension diagnosis using a multivariable test $(P<.001)$. Obesity also significantly factored into 


\section{TABLE 4. Predictors of Subsequent Hypertension Diagnosis}

Analysis of Hypertension Diagnosis Made $(n=1400)$ vs No Hypertension Diagnosis Made $(n=1848)$

\begin{tabular}{|c|c|c|c|c|c|c|}
\hline \multirow{3}{*}{ Variable } & \multirow{2}{*}{\multicolumn{3}{|c|}{ Bivariate }} & & & \\
\hline & & & & \multicolumn{3}{|c|}{ Multivariable } \\
\hline & $\mathbf{R R}$ & $95 \% \mathrm{Cl}$ & $P$ Value & $\mathbf{R R}$ & $95 \% \mathrm{Cl}$ & $P$ Value \\
\hline \multicolumn{7}{|l|}{ Sex } \\
\hline Male & 1.0 & & & 1.0 & & \\
\hline Female & 0.90 & $0.83-0.97$ & .008 & 0.99 & $0.91-1.09$ & .894 \\
\hline \multicolumn{7}{|l|}{ Race } \\
\hline White & 1.0 & & & & & \\
\hline Black/African American & 1.32 & $1.19-1.46$ & $<.001$ & 1.39 & $1.24-1.55$ & $<.001$ \\
\hline \multicolumn{7}{|l|}{ Age, y } \\
\hline $18-35$ & 1.0 & & & 1.0 & & Reference \\
\hline $36-49$ & 1.54 & $1.35-1.77$ & $<.001$ & 1.53 & $1.32-1.77$ & $<.001$ \\
\hline $50-64$ & 1.82 & $1.60-2.08$ & $<.001$ & 1.95 & $1.70-2.24$ & $<.001$ \\
\hline$\geq 65$ & 1.74 & $1.46-2.06$ & $<.001$ & 2.16 & $1.79-2.60$ & $<.001$ \\
\hline \multicolumn{7}{|l|}{ Health insurance } \\
\hline Insured & 1.0 & & & 1.0 & & \\
\hline Uninsured & 1.25 & $1.16-1.36$ & $<.001$ & 1.33 & $1.22-1.46$ & $<.001$ \\
\hline \multicolumn{7}{|l|}{ Housing } \\
\hline Home & 1.0 & & & & & \\
\hline Housing insecure & 0.90 & $0.71-1.13$ & .357 & 0.88 & $0.69-1.14$ & .338 \\
\hline \multicolumn{7}{|l|}{ Obesity (BMl in kg/m²) } \\
\hline Normal weight (BMl <25) & 1.0 & & & 1.0 & & \\
\hline Overweight (BMI 25-29.9) & 1.13 & $0.99-1.29$ & .073 & 1.07 & $0.92-1.23$ & .392 \\
\hline Obesity (BMI $\geq 30$ ) & 1.28 & $1.14-1.43$ & $<.001$ & 1.42 & $1.25-1.61$ & $<.001$ \\
\hline
\end{tabular}

Abbreviations: $\mathrm{BMI}$, body mass index; $\mathrm{Cl}$, confidence interval; $\mathrm{RR}$, risk ratio.

subsequent hypertension diagnoses for HIPS patients. Patients with obesity were $42 \%$ more likely to be diagnosed with hypertension than healthy weight patients when controlling for other variables $(P<.001)$.

A visual summary of characteristics that describe the HIPS population and predictive characteristics for each subgroup is shown in Figure 3.

\section{DISCUSSION}

Undiagnosed hypertension is a problem in the United States. These analyses reveal factors that may lead to disproportionate rates of undiagnosed hypertension in certain populations.

\section{Patients identified by the HIPS algorithm as potentially undiagnosed for hypertension}

Given that females comprised $62.3 \%$ of the adult patients across the 3 health centers, it was not surprising that $61.9 \%$ of the patients identified by the HIPS algorithm were females. In contrast, racial differences among HIPS patients represent a significant difference from the overall population served by these centers. Blacks/African Americans represent $73.0 \%$ of patients identified versus $58.7 \%$ of patients overall in the participating health center population. This finding is consistent with national data from the 2015-2016 NHANES Survey, ${ }^{14}$ which indicate that the prevalence of hypertension among non-Hispanic black adults is proportionately higher than among non-Hispanic whites $(40.3 \%$ vs $27.8 \%)$.

Other research has shown that social context, not race, underlies higher hypertension rates among the black/African American population. ${ }^{24,25}$ Specifically, hypertension was found to be negatively associated with neighborhood affluence. In one study, significantly more blacks/African Americans were found to meet the clinical criteria for hypertension; however, after adjusting for neighborhood affluence, racial differences in rates of hypertension vanished. ${ }^{25}$ A retrospective study by Thorpe et $\mathrm{al}^{24}$ found that social and environmental factors explained a considerable amount of race difference 


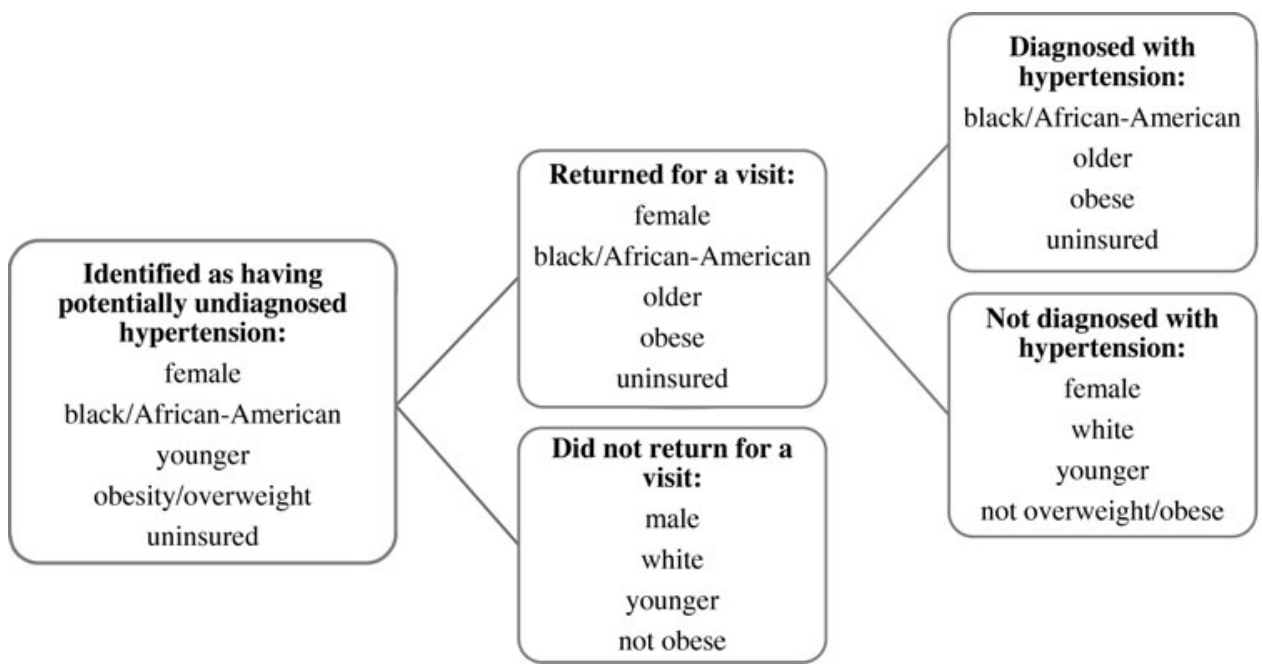

Figure 3. Summary of characteristics of those identified by the HIPS algorithm and subgroups. HIPS indicates hiding in plain sight.

in hypertension. Thus, it is possible that poverty and other social determinants of health play an important role in the race disparities we found in hypertension diagnoses.

In addition to racial differences, our study found that younger people may be at higher risk of having their hypertension remain undiagnosed. Most health centers serve a younger population of patients, with the proportion aged 18 to 64 years in the 3 participating health centers representing $90.6 \%$ of their adult patients. ${ }^{26}$ Patients aged 18 to 64 years identified by the HIPS algorithm comprised $92.0 \%$ of patients, a small but potentially meaningful difference. Of the HIPS patients in our study who returned for a visit, those aged 18 to 35 years comprised $28 \%$ of the patients diagnosed with hypertension, which is over 3 times higher than the NHANES national hypertension prevalence rate of $7.5 \%$ for this age group. This discrepancy suggests younger people's blood pressure may be more likely to be dismissed when they have high readings at office visits, a finding consistent with previous research. ${ }^{13}$

Insurance status also factored into the characteristics of those identified by the HIPS algorithm. Nearly half of the HIPS patients were uninsured, while the average uninsured rate at the participating sites was $32.5 \%$ for adults. Similar to younger age, one explanation for the higher percentage of uninsured patients found with potentially undiagnosed hypertension is that those without insurance may historically have been more likely to have their elevated readings disregarded and/or hypertension diagnoses delayed when presenting with elevated blood pressure readings at office visits. Previous research has indicated that patient insurance status has influenced clinician decision making and altered clinical management. ${ }^{27}$ Thus, this higher-thanexpected percentage of uninsured patients identified by the HIPS algorithm could point to disparities in care for these patients even at health centers where patients are cared for regardless of ability to pay.

The majority of patients identified by the HIPS algorithm were classified as having obesity $(55.0 \%)$. By comparison, $31.7 \%$ of adults in Missouri are obese overall and $38.6 \%$ of black/African American adults in Missouri are classified as obese. ${ }^{28}$ The fact that over half of HIPS patients were classified as having obesity is not surprising, given the known link between obesity and hypertension. ${ }^{29}$ What might be considered perplexing is why so many patients with obesity remained potentially undiagnosed for hypertension, only to be identified by our algorithm, given the general knowledge that obesity is a risk factor for hypertension.

One possible explanation is that people with obesity tend to have other comorbidities, including osteoarthritis, chronic back pain, gallbladder disease, asthma, diabetes, and cancer, ${ }^{30}$ and perhaps addressing one or more of these other conditions took precedence over diagnosing and managing hypertension. Standardized treatment protocols that cue abnormal blood pressure values to be addressed even when blood pressure evaluation is not the primary cause of the patient visit may help promote timely hypertension diagnosis. Obesity was the only comorbidity collected in the HIPS data set. Exploring whether complex patients are more likely to have undiagnosed hypertension could be an important future direction. 
Even though all patients were identified using the same clinical criteria (either 2 systolic blood pressure [SBP] measurements between 140 and $159 \mathrm{~mm} \mathrm{Hg}$ or diastolic blood pressure [DBP] measurement between 90 and $99 \mathrm{~mm} \mathrm{Hg}$ or 1 SBP measurement $\geq 160 \mathrm{~mm} \mathrm{Hg}$ or DBP measurement $\geq 100 \mathrm{~mm} \mathrm{Hg}$ ), various patient characteristics predicted whether or not a patient had a return visit where blood pressure was assessed and whether or not they received a hypertension diagnosis.

\section{Factors associated with HIPS patients returning for a follow-up visit}

HIPS patients who returned for a visit can be described as more likely to be female, classified as having obesity, older ( $\geq 50$ years), and black/African American. A longitudinal analysis by Green and Pope $^{31}$ showed that sex is an independent predictor for use of medical services. Thus, the finding that females were more likely than males to have had previously scheduled appointments and/or to respond to prompts to have their blood pressure evaluated is unsurprising. The propensity for patients with obesity to return for a visit in the followup time frame is also not surprising; people with obesity are more likely to have other health conditions that might lead them to seek medical care. ${ }^{30}$ Moreover, advanced age is an established predictor of increased health care visits. ${ }^{32}$

What is less clear is why HIPS patients who are black/African American were more likely to have a visit in the follow-up period. Data from the 2016 National Health Interview Survey indicate no meaningful racial differences in regularity of interaction with a health care provider. ${ }^{32}$ In addition, mistrust of the US health care system by blacks/African Americans as a result of historic mistreatment is well documented, ${ }^{33}$ a factor that could decrease health care-seeking behavior, not increase it. That our findings run counter to this evidence suggests there may be something unique about health centers compared with other health care delivery organizations that have fostered greater trust among blacks/African Americans both in seeking regular health care and responding to outreach to have their blood pressure checked.

HIPS patients who did not return for a visit where blood pressure was assessed were more likely to be white, male, between the ages of 18 and 35 years, insured, and either healthy weight or overweight but not diagnosed with obesity. These characteristics align with previous research that found males between the ages of 15 and 50 years seldom seek preventive care services, irrespective of insurance status. ${ }^{20}$ Taken holistically, understanding which patients meet the clinical criteria for hyper- tension but are less likely to return for a visit is important so that more effective behavior change interventions that specifically target these individuals can be deployed.

\section{Factors associated with HIPS patients receiving a hypertension diagnosis}

Overall, nearly half $(43 \%)$ of patients identified by the HIPS algorithm were diagnosed with hypertension on follow-up in this study. This finding reinforces the importance of deliberate efforts to identify patients whose current and/or past blood pressure measurements meet the clinical criteria for hypertension to bring them back for assessment. Specific factors were also associated with HIPS patients receiving a hypertension diagnosis.

Age remained a strong predictor of whether a HIPS patient received a formal hypertension diagnosis. Perhaps this is the case because these older patients had risk factors other than obesity that were not assessed. However, as discussed previously, it is also possible that expectations about which patients should have hypertension may be creating bias toward diagnosing older patients more quickly, while taking a less aggressive approach with younger patients.

Similar to age, when other factors were controlled for, black/African American HIPS patients were significantly more likely to be diagnosed with hypertension than white patients. This cannot be easily explained by the higher known prevalence of diagnosed hypertension among black/African American patients, since the pool of patients identified all met the same clinical criteria described previously (HIPS algorithm). It is possible there are other factors clinicians considered beyond those for which we controlled; for example, other comorbidities, stress levels, and other social determinants of health. However, these findings also suggest the possibility of diagnosing behavior occurring based on a certain demographic profile. Provider expectations for who should receive a diagnosis may reinforce actual diagnosis of black/African American patients at higher rates than whites and older patients at higher rates than younger patients. The known higher prevalence of hypertension in older, black/African American people can lead providers to react more quickly to elevated blood pressure readings and increase the likelihood of the provider making a diagnosis. ${ }^{6,14,15,32}$

Being overweight or having obesity, known risk factors for hypertension, ${ }^{30}$ also predicted hypertension diagnosis. Appropriately diagnosing these patients is certainly positive. The question is whether subjective human decision-making layered onto an objective use of data to identify patients with 
hypertension is leaving some patients at risk for cardiovascular events linked to untreated hypertension-healthy-weight people, similar to the younger patients and white patients discussed earlier, may be at risk for remaining undiagnosed for hypertension.

Significantly more uninsured patients were subsequently diagnosed with hypertension. One possibility for this discrepancy might be that, in the context of a project focused on addressing undiagnosed hypertension, providers were more likely to diagnose patients appropriately based on objective clinical criteria whereas previously uninsured patients may have been less likely to be diagnosed for hypertension. It warrants further exploration to understand more fully whether bias regarding patients' insurance status may cause them to receive differential care for chronic disease in circumstances where there is not explicit focus on diagnosing patients who meet the clinical definition of hypertension. Moreover, understanding the role of nonclinical factors in both increasing rates of hypertension in certain populations and how these factors influence hypertension diagnosis are important areas for further study.

HIPS patients who are younger, white, female, and/or who are healthy weight were less likely to be diagnosed with hypertension. While some of these patients were likely ruled out for hypertension because they had normal blood pressure measurements at their return visit, others were missed and remain hiding in plain sight with hypertension. Some of these people may have been categorized as having white coat hypertension-when a patient has elevated blood pressure values in a clinical setting, but normal blood pressure values elsewhere. ${ }^{34}$ White coat hypertension is difficult to evaluate accurately without automated office blood pressure (AOBP) machine use or out-ofoffice measurements. ${ }^{35}$ No evidence currently exists to suggest that younger, white, female patients who are healthy weight have white coat effect at higher rates than people of other ages, races, and weights. Thus, it is unlikely that this phenomenon explains the higher proportion of patients in this group who remained undiagnosed. It is possible that providers could be taking a more conservative "wait-and-see" approach with patients who do not fit the profile of who they expect to have hypertension. This potential bias is based on existing higher hypertension prevalence rates among patients who are older, African American, and male. ${ }^{6,14,15,32}$

\section{Future directions}

Pairing data-driven interventions to identify undiagnosed hypertension with AOBP machine usage and/or self-measured blood pressure monitor- ing (SMBP) may help reduce bias and subjective decision-making in hypertension diagnosis; since AOBP machines and SMBP both yield more accurate patterns of blood pressure measurements over time than traditional in-office measurements, these can help accelerate appropriate diagnosis and treatment for true hypertension. ${ }^{5}$

Future studies could explore the role of nonclinical factors in rates of hypertension in certain populations and how these factors may influence hypertension diagnosis. Specifically, additional studies could examine the role of social determinants of health in race disparities in hypertension prevalence. Moreover, the role of clinicians' expectations in timely hypertension diagnosis could also be explored; in particular, the propensity for clinicians to move to a diagnosis more quickly in patients who they believe are more likely to have hypertension.

\section{Limitations}

The HIPS algorithm did not distinguish between undocumented hypertension and undiagnosed hypertension. While the algorithm excluded patients with hypertension diagnoses documented on their EHR's problem list or within an encounter, it could have identified patients as having undiagnosed hypertension who may be receiving treatment but whose diagnosis was not documented in 1 of these 2 places. However, prior research has found that treatment for hypertension is strongly associated with having a documented hypertension diagnosis..$^{10}$ Thus, it is unlikely that many in the HIPS sample who had no diagnosis of hypertension in their EHR were being treated for the disease. Further, there is no reason to suspect that this situation of undocumented hypertension was more or less likely among any demographic group.

In addition, we do not know which patients had a single very elevated blood pressure reading versus 2 moderately elevated blood pressure readings (or some combination) to meet the criteria that identified them as potentially undiagnosed for hypertension and we do not know what their actual blood pressure values were, either for identification by the HIPS algorithm or upon follow-up assessment.

We do not know whether other comorbidities, except obesity, may have impacted provider inclination to diagnose versus not diagnose in older patients in particular. Additionally, poverty level likely played a role in return rates and hypertension diagnosis, but could not be analyzed due to insufficient data. Poverty level may further translate to health determinants like food insecurity, stress, and lack of regular employment, which may be confounding factors in understanding differences uncovered in this study. 
Finally, health centers represent an underserved population. The findings from this project may not be generalizable to other patient populations.

\section{CONCLUSIONS}

This study provided an important first step toward understanding the characteristics of patients with undiagnosed hypertension, including those who met the clinical criteria for hypertension using an algorithm and those who were formally diagnosed with hypertension. The variation in the attributes of those identified by the HIPS algorithm and the subgroups based on return visit and hypertension diagnosis reveals important differences that are opportunities for future tailored intervention strategies. In addition, differences illuminated between these groups indicate a need to address possible biases toward diagnosing versus delaying/not diagnosing certain types of patients-important knowledge and awareness that can be incorporated into care team training to reduce disparities in hypertension identification and care. Finally, our study drew further attention to the importance of understanding the role of social context in hypertension among blacks/African Americans.

\section{REFERENCES}

1. Benjamin EJ, Virani SS, Callaway CW, et al.; American Heart Association Council on Epidemiology and Prevention Statistics Committee and Stroke Statistics Subcommittee. Heart disease and stroke statistics-2018 update: a report from the American Heart Association. Circulation. 2018;137(12):e67-492.

2. Yoon PW, Gillespie CD, George MG, Wall HK. Control of hypertension among adults - National Health and Nutrition Examination Survey, United States, 2005-2008. MMWR Suppl. 2012;61(2):19-25.

3. Centers for Disease Control and Prevention. Vital signs: avoidable deaths from heart disease, stroke, and hypertensive disease-United States, 2001-2010. MMWR Morb Mortal Wkly Rep. 2013;62(35):721-727.

4. Esposito D, Bagchi AD, Verdier JM, Bencio DS, Kim MS. Medicaid beneficiaries with congestive heart failure: association of medication adherence with healthcare use and costs. Am J Manag Care. 2009;15:437-445.

5. Whelton PK, Carey RM, Aronow WS, et al. 2017 ACC/ AHA/AAPA/ABC/ACPM/AGS/APhA/ASH/ASPC/NMA

PCNA guideline for the prevention, detection, evaluation, and management of high blood pressure in adults: a report of the American College of Cardiology/American Heart Association Task Force on Clinical Practice Guidelines. Hypertension. 2018;71:e13-e115. http://hyper.ahajournals. org/content/early/2017/11/10/HYP.0000000000000065.

6. Wall HK, Ritchey MD, Gillespie C, Omura JO, Jamal A, George MG. Vital signs: prevalence of key cardiovascular disease risk factors for million hearts 2022-2011-2016. MMWR Morb Mortal Wkly Rep. 2018;67(35):983-991.

7. Wall HK, Hannan JA, Wright JS. Patients with undiagnosed hypertension: hiding in plain sight. JAMA. 2014;312:19731974.

8. Baus A, Hendryx M, Pollard C. Identifying patients with hypertension: a case for auditing electronic health record data. Perspect Health Inf Manag. 2012;9:1e.
9. Rakotz MK, Ewigman BG, Sarav M, et al. A technologybased quality innovation to identify undiagnosed hypertension among active primary care patients. Ann Fam Med. 2014;12(4):352-358.

10. Banerjee D, Chung S, Wong EC, Wang EJ, Stafford RS, Palaniappan LP. Underdiagnosis of hypertension using electronic health records. Am J Hypertens. 2012;25(1):97102.

11. Mishuris RG, Yoder J, Wilson D, Mann D. Integrating data from an online diabetes prevention program into an electronic health record and clinical workflow, a design phase usability study. BMC Med Inform Decis Mak. 2016;16:88.

12. Meador M, Osheroff JA, Reisler B. Improving identification and diagnosis of hypertensive patients hiding in plain sight (HIPS) in health centers. Jt Com J Qual Patient Saf. 2018;44(3):117-129.

13. Johnson HM, Thorpe CT, Bartels CM, et al. Undiagnosed hypertension among young adults with regular primary care use. J Hypertens. 2014;32(1):65-74. doi:10.1097/HJH.0000000000000008.

14. Fryar CD, Ostchega Y, Hales CM, Zhang G, KruszonMoran D. Hypertension Prevalence and Control Among Adults: United States, 2015-2016. NCHS Data Brief, no 289. Hyattsville, MD: National Center for Health Statistics; 2017. https://www.cdc.gov/nchs/products/databriefs/ db289.htm. Accessed April 30, 2018.

15. Whelton PK. The elusiveness of population-wide high blood pressure control. Annu Rev Public Health. 2015; 36:109-130.

16. Kennard L, O'Shaughnessy KM. Treating hypertension in patients with medical comorbidities. BMJ. 2016;352:1101

17. Hadley J. Sicker and poorer-the consequences of being uninsured: a review of the research on the relationship between health insurance, medical care use, health, work, and income. Med Care Res Rev. 2003;60(2 suppl):3S75S; discussion 76S-112S.

18. Ayania JZ, Zaslavsky AM, Weissman JS, Schneider EC, Ginsburg JA. Undiagnosed hypertension and hypercholesterolemia among uninsured and insured adults in the Third National Health and Nutrition Examination Survey. Am J Pub Health. 2000;93(12):2051-2054.

19. DeVoe JE, Fryer GE, Phillips R, Green L. Receipt of preventive care among adults: Insurance status and usual source of care. Am J Pub Hlth. 2003;5:786-791. https://ajph. aphapublications.org/doi/abs/10.2105/AJPH.93.5.786.

20. Alt RL. Where the boys are not: a brief overview of male preventive health. Wisconsin Med J. 2002;101(4):22-27.

21. Gil-Guillén V, Orozco-Beltrán D, Pérez RP, et al. Clinical inertia in diagnosis and treatment of hypertension in primary care: quantification and association factors. Blood Press. 2009;19(1):3-10. doi:10.3109/08037050903350762.

22. Goodman D, Ogrinc G, Davies L, et al. Explanation and elaboration of the SQUIRE (Standards for Quality Improvement Reporting Excellence) Guidelines, V.2.0: examples of SQUIRE elements in the healthcare improvement literature. BMJ Qual Saf. 2016;25:e7. doi:10.1136/bmjqs2015-004480.

23. Zou G. A modified Poisson regression approach to prospective studies with binary data. Am J Epid. 2004; 159(7):702-706. doi:10.1093/aje/kwh090.

24. Thorpe RJ, Brandon DT, LaViest TA. Social context as an explanation for race disparities in hypertension: findings from the Exploring Health Disparities in Integrated Communities (EHDIC) Study. Soc Sci Med. 2008;67(10):16041611.

25. Morenoff JD, House JS, Hanson BB, Williams DR, Kaplan GA, Hunte HE. Understanding social disparities in hypertension prevalence; awareness, treatment, and control: 
the role of neighborhood context. Soc Sci Med. 2007; 65(9):1853-1866.

26. Health Resources and Services Administration, Bureau of Primary Health Care. Uniform data system: 2016 UDS tables. https://bphc.hrsa.gov/uds/datacenter.aspx?q=d. Accessed April 30, 2018.

27. Meyers DS, Mishori R, McCann J, Delgado J, O'Malley AS, Fryer E. Primary care physicians' perceptions of the effect of insurance status on clinical decision making. Ann Fam Med. 2006;4(5):399-402.

28. Robert Wood Johnson Foundation and Trust for America's Health. The State of Obesity: Better Policies for a Healthier America. https://stateofobesity.org/states/mo/ Published August 2017. Accessed May 29, 2018.

29. Richard N Re. Obesity-related hypertension. Ochsner J. 2009;9(3):133-136.

30. Guh DP, Zhang W, Bansback N, Amarsi Z, Birmingham $\mathrm{CL}$, Anis $\mathrm{AH}$. The incidence of co-morbidities related to obesity and overweight: a systematic review and metaanalysis. BMC Public Health. 2009;9:88. doi:10.1186/ 1471-2458-9-88.
31. Green CA, Pope CR. Gender, psychosocial factors and the use of medical services: a longitudinal analysis. Soc Sci Med. 1999;48(10):1363-1372.

32. Centers for Disease Control and Prevention. Summary Health Statistics: National Health Interview Survey, 2016, Table A-18a. https://ftp.cdc.gov/pub/Health_Statistics/ NCHS/NHIS/SHS/2016_SHS_Table_A-18.pdf. Accessed August 13, 2018.

33. Kennedy BR, Mathis CC, Woods AK. African Americans and their distrust of the health care system: healthcare for diverse populations. J Cult Div. 2007;14(2): 56-60.

34. de la Sierra A. Definition of white coat hypertension: ambulatory blood pressure, self-measured blood pressure, or both? Hypertension. 2013;62:16-17.

35. Rakotz M. The Importance of Measuring Blood Pressure Accurately. American Medical Association. http:// greatplainsqin.org/wp-content/uploads/2016/11/TheImportance-of-Measuring-BP-Accurately-Handout-Nov10.pdf. Presented November 10, 2016. Accessed April 30, 2018. 